\title{
Network of multi-omics reveals the mechanism of russeting under rainwater in pears
}

\author{
Chun-hui Shi ${ }^{1}$, Xiao-qing Wang ${ }^{1}$, Yu-xing Zhang ${ }^{2}$, and Jun Luo ${ }^{1}$ \\ ${ }^{1}$ Shanghai Academy of Agricultural Sciences \\ ${ }^{2}$ Hebei Agricultural University
}

May 11, 2020

\begin{abstract}
Russeting occurs via the epidermal suberin accumulation model in the phenol-ester protection system in pears, especially under excessive rainfall. To clarify the role of rain in the russeting mechanism, we applied multi-omics (transcriptome, metabolome, and proteome) integrated analysis from three colored pear cultivars grown under two rainfall conditions, including a no rain control (C) and a rain-abundant (R) treatment. Broad alterations occurred in 29 overlapping differentially expressed genes (DEGs) involved in the response to stress and secondary metabolite biosynthesis. Three overlapping differentially expressed proteins (DEPs) and seven differentially expressed metabolites (DEMs) were identified among the three comparison groups. Cross-comparison of mRNA and protein data revealed co-expression of DEGs and DEPs. Suberin and phenylpropane were markedly enhanced, while cutin biosynthesis and fatty acid elongation were depressed in russet pears under R. In russet pears, the triacontane associated with wax composition was abruptly missing. Moreover, The PpyHHT1 (103966555) gene and encoded protein HHT1 (694406379), which acts as a 'bridge' between phenol- and ester- polymerization, had significantly upregulated expression. Collectively, we provide a comprehensive model of the molecular russeting mechanism, which provides powerful insight into russeting and shows the plasticity of plant defenses to cope with the harsh natural environment.
\end{abstract}

\section{Acknowledgements}

This study was financially supported by Research and Demonstration of High-efficiency Production Technology in Green Pear Orchard (SAS2019 1-3). In addition, we thank the anonymous reviewers for their valuable comments regarding the manuscript.

Running Head: Multi-omics reveal russeting mechanism in pear

TITLE: Network of multi-omics reveals the mechanism of russeting under rainwater in pears

Chun-hui Shi ${ }^{1,2}$, DAg; Xiao-qing Wang ${ }^{1}$, MAg; Yu-xing Zhang ${ }^{2}$, DAg; Jun Luo ${ }^{{ }^{*}}$, BAg

${ }^{1}$ Forest \& Fruit Tree Research Institute, Shanghai Academy of Agricultural Sciences, Shanghai City, 201403 China

${ }^{2}$ College of Horticulture, Hebei Agricultural University, Baoding City, Hebei Province, 071001 China

*Corresponding authors: Jun Luo

E-mail: zuowusuo@163.com Tel: +86-021-52235473

Address: Forest \& Fruit Tree Research Institute, Shanghai Academy of Agriculture Sciences, 1000 Jinqi Road, Fengxian District, Shanghai City, 201403 China

Abstract 
Russeting occurs via the epidermal suberin accumulation model in the phenol-ester protection system in pears, especially under excessive rainfall. To clarify the role of rain in the russeting mechanism, we applied multi-omics (transcriptome, metabolome, and proteome) integrated analysis from three colored pear cultivars grown under two rainfall conditions, including a no rain control $(\mathrm{C})$ and a rain-abundant $(\mathrm{R})$ treatment. Broad alterations occurred in 29 overlapping differentially expressed genes (DEGs) involved in the response to stress and secondary metabolite biosynthesis. Three overlapping differentially expressed proteins (DEPs) and seven differentially expressed metabolites (DEMs) were identified among the three comparison groups. Cross-comparison of mRNA and protein data revealed co-expression of DEGs and DEPs. Suberin and phenylpropane were markedly enhanced, while cutin biosynthesis and fatty acid elongation were depressed in russet pears under R. In russet pears, the triacontane associated with wax composition was abruptly missing. Moreover, The PpyHHT1 (103966555) gene and encoded protein HHT1 (694406379), which acts as a 'bridge' between phenol- and ester- polymerization, had significantly upregulated expression. Collectively, we provide a comprehensive model of the molecular russeting mechanism, which provides powerful insight into russeting and shows the plasticity of plant defenses to cope with the harsh natural environment.

Keywords: russeting, suberin, phenol-ester, sand pear, exocarp, rain-shelter, rainfall, muti-omics, phenylpropane, rainwater.

\section{Introduction}

Fruit color, a key indicator for evaluating quality, is a major factor affecting consumer purchases (Gamble et al., 2006). Russet and semi-russet (partial russet) color is unique to sand pear (Pyrus pyrifolia Nakai.), which is a species native to Eastern Asia, including China, Japan, and Korea. In recent years, the cultivation area has spread to Australia, America, and Europe and it is still spreading. Unlike common fruit colors, russet is not determined by anthocyanins, but by suberin lamellae accumulation when there is a cuticle defect on the green exocarp (Inoue et al., 2006; Wang et al., 2016). The tissue structure balance between suberin lamellae and stratum corneum affects pear coloration. Although both tissues have a similar function as a periderm to resist pressure from the harsh environment, the suberin lamellae is more plastic under environmental stress (Laraet al. , 2015; Markus and Lukas, 2001). Emerging evidence has indicated that heavy rainfall may be one of the most important factors inducing suberin accumulation (Lara et al. , 2015). In preliminary studies, we observed a sharp increase in lignin, the main compound comprising the russet exocarp under excessive rainfall (Shi et al., 2019a). This affects phenylalanine lignin monomer synthesis and defense metabolism by regulating the corresponding proteins. Here, we further study russeting by creating a comprehensive network using multi-omics (transcriptomics, proteomics, and metabolomics) data.

Suberin is the main component of suberin lamellae; it is polymerized from compounds such as glycerol, fatty acids (FAs), and phenols, as well as suberin-related waxes, lignin, tannins, and other phenolics (Franke and Schreiber, 2007; Pollard et al., 2008). It is unknown how the suberin poly-aliphatic domain (SPAD) and suberin poly-phenolic domain (SPDD) assemble out of the cell wall after small molecules in the cytoplasm cross the plasma membrane. Waxy components are present in both the cuticle layer and the suberin lamellae. Suberin is a cutin analog polymer and is primarily composed of carbon chains $\left(\mathrm{C}_{16}-\mathrm{C}_{26}\right)$, long chain fatty acids (LCFAs), and phenolics linked with alkyl ferulate-waxes, whereas the cuticle layer is rich in $\mathrm{C}_{16}-\mathrm{C}_{18}$ fatty acids (Molina et al., 2006; Cohen et al., 2017). Previous studies have indicated that the proportion of lignin, cellulose, and hemicellulose in the russet pear were significantly higher than in the green bud pear, while the proportion of pectin polysaccharides and long-chain fatty acids were higher in the green pear. In pears and apples, the molecular mechanism of suberin biosynthesis was studied based on transcriptional analysis and masses of candidate genes were identified. The genes encoding COMT (Caffeic acid 3-O-methyltransferase), CCOAOMT (Caffeoyl CoA-O-methyltransferase), CCR (CinnamoylCoA reductase), CAD (Cinnamyl alcohol dehydrogenase), and POD (Peroxidase) in lignin biosynthesis and the genes encoding BAHD family acyltransferase, HHT ( $\omega$-hydroxypalmitate-O-feruloyl transferase), GPAT (Glycerol phosphate acyltransferase) faminly, KCS (3-ketoacyl-CoA synthase), ATP binding cassette (ABC) transporter, and MYB93-like transcription factor in suberin biosynthesis were considered the key genes for suberized tissue deposition (Wang et al ., 2014a; Lashbrooke et al ., 2015; Legay et al., 2015; Hou et al. , 
2018). However, much less is known about the specific mechanism by which rainwater affects this complex biosynthesis pathway and a detailed cork synthesis and transmembrane assembly model is needed.

High-throughput omics techniques, such as transcriptomic, proteomic, metabolomic, and genomic analyses, have been extensively employed in the study of plant resistance to stress (Jia et al., 2019). Wang et al (2014b) and Wei et al (2016) have identified a set of genes related to the formation of the cuticle and suberin lamellae through RNA-seq technology. Despite global data on the involvement of suberin in russeting, there have been no reports that rainwater promotes russeting.

To explored how rainfall influences russeting through suberin deposit-related pathways, we mapped a connection network using transcriptome, proteome, and metabolome correlation analysis from under rainwater treatment in three colored pear cultivars: russet 'Zaoshengxinshui', semi-russet 'Cuiguan', and green 'Cuiyu'. By comparing the DEGs, DEPs, and DEMs of exocarp with and without rain, we apply a novel targeted protein technology (parallel reaction monitoring, PRM) (Martinez et al.,2017; Miao et al., 2018), targeted metabolites technology (LC-MS), and targeted gene technology (quantitative real-time PCR). These techniques may be used to validate the reliability of proteins, genes, and metabolites, leading to core russeting process nodes affected by rainfall. Our findings provide a rich resource for in-depth insights into the mechanism of russeting.

\section{Results}

\section{Fruit micromorphology of three cultivars}

Russeting is essentially the accumulation of suberin lamellae on the exocarp. The russeting of sand pears is aggravated by rain, with suberin lamellae of the semi-russet exocarp (partial russet) showing an enlarged area and that of russet exocarp becoming thicker (Fig. S1). Russet and semi-russet pears had significant differences in the color of their fruits' skins after $\mathrm{R}$ treatment (Fig. 1A). Specifically, in the semi-russet exocarp, the russet index was upregulated in the R-treated group when compared to the C-treated group $(29.62 \%)$. Similarly, the russet exocarp after R treatment had a thicker suberin lamellae $(0.080 \mathrm{~mm})$ than the C-treated exocarp $(0.043 \mathrm{~mm})$ (Figs. 1C and 1D). Moreover, in the R group, the cuticle of the fruit epidermis had extensive cracks, the most severe of which were accompanied by shedding, epidermal damage, and exocarp formation. Although there were cracks in the cuticle of the pear epidermis in the C-treated group, the fruit surface did not form a large area of suberin lamellae accumulation (Fig. 1B). After the green pear was treated with $\mathrm{R}$, the stratum corneum cracked but no plugged tissue formed at the crack.

\section{Metabolome profiling}

A total of 3391 metabolites were identified (3135 identified by LC-MS and 256 by GC-MS). PCA analysis revealed a clear separation between treatments and controls (Figs. 2A and 2B). Combining the results of PLS-DA modeling and student's t-test, 363, 320, and 84 DEMs were identified between semi-russet pear (CG-R vs CG-C), russet pear (ZS-R vs ZS-C), and green pear (CY-R vs CY-C), respectively (Fig. 2C). After comparative analysis, 9 overlapped DEMs were identified between all cultivars and treatments (Fig. 2C). Subsequently, KEGG enrichment analysis showed only lutein contained the KEGG ID (C08601) and it was mainly enriched in biosynthesis of terpenoids and steroids and secondary metabolite biosynthesis.

The metabolites associated with suberin lamellae formation in exocarp russeting were studied further. Significant differences were found between the R- and C-treated groups in 84 DEMs, clustered into 8 functional groups (Fig. 2D and Table S1). Among the 9 phenolic substances detected, all phenols exhibited higher levels in the R-treated group than in the C-treated group in russet and semi-russet exocarp, while these levels were lower in green exocarp. Most remarkably, $( \pm)$-catechin, caffeoyl cycloartenol, and 3-O-p-transcoumaroylalphitolic acid were significantly increased. Cinnamic acid and methyl 3,4,5-trimethoxycinnamate [arabinosyl-(1->3)-[glucosyl-(1->6)]-glucosyl] ester significantly increased in the russet pear under $\mathrm{R}$ treatment. In contrast, the content of phenylalanyl-tryptophan and 3-O-p-trans-coumaroylalphitolic acid were significantly decreased by factors of 3.94 and 1.81, respectively, in green pear. Suberin and cutin are glycerolbased polymers with complex chemical compositions, with many FAs accounting for a large proportion of the 
suberin (Heng et al., 2014; Pollard et al., 2008). A total of 9 LCFAs $\left(\mathrm{C}_{18}\right)$ were identified, mostly consisting of octadecatrienoic acids ( $\mathrm{C}_{18}$ unsaturated fatty acids), all of which exhibited higher levels in the semi-russet exocarp under $\mathrm{R}$ treatment. With consistent and significant increases, 3E, 9Z, 12Z-octadecatrienoic acid, and pinolenic acid (5Z, 9Z, 12Z-octadecatrienoic acid) exhibited higher levels in russet and semi-russet exocarp, while these FAs did not change in the green exocarp.

Absolute quantitative analysis of the above DEMs by LC-MS showed that the quantities of complex phenolic polymer-lignin in the R-treated group were higher than those in the C-treated group of russet and semirusset exocarps $(\mathrm{P}<0.05)$. The small molecule phenol substance epicatechin, palmitic acid $\left(\mathrm{C}_{16: 0}\right)$, VLCFAs $\left(\mathrm{C}_{20}-\mathrm{C}_{24}\right)$ [including eicosadienoic acid $\left(\mathrm{C}_{20}\right)$ and gadoleic acid $\left(\mathrm{C}_{20}\right)$ ], significantly increased in the R-treated group when compared to those in the $\mathrm{C}$-treated group of russet and semi-russet exocarps $(\mathrm{P}<0.01)$ (Fig. 2E). Erucic acid $\left(\mathrm{C}_{22}\right)$ and nervonic acid $\left(\mathrm{C}_{24}\right)$ increased significantly in the semi-russet exocarp by 2.02 -fold and $68.6 \%$, respectively, while the contents of VLCFAs in the green exocarp were significantly reduced $(46.17 \%)$ in the R-treated group. Similarly, the proportion of LCFAs $\left(\mathrm{C}_{18}\right)$, such as elaidic acid, linoleic acid, oleic acid, and $\gamma$-Linoenic acid, were significantly increased in the semi-russet exocarp.

\section{Transcriptional analysis}

Transcriptome analysis was performed with the same samples used for the metabolome analysis. After quality assessment and data filtering, a total of 1718 (including 988 upregulated and 730 downregulated), 803 (including 424 upregulated and 379 downregulated), and 2545 (including 1831 upregulated and 714 downregulated) transcripts were differentially expressed in the semi-russet pear (CG-R vs CG-C), russet pear (ZS-R vs ZS-C), and green pear (CY-R vs CY-C), respectively. Following comparative analysis, the overlapped DEGs between different groups were obtained. A total of 29 DEGs were found to be differentially expressed among all cultivars and treatments (Fig. 3A and Table S2). Using GO enrichment analysis, overlapping molecular functions of the DEGs identified in the three groups were classified into categories, such as biological process and cellular component, among which the response to cell wall stress were the dominant functions (Fig. 3B and Table S3). Additionally, the results of KEGG pathway analysis showed that the DEGs among all cultivars and treatments were overlapping in three enriched pathways, including biosynthesis of secondary metabolites, biosynthesis of cutin, suberin and wax, and biosynthesis of carotenoid (Fig. 3C and Table S4).

In the R-treated group, the suberin lamellae accumulated in russet and semi-russet exocarps, but there was almost no accumulation in the green exocarp. These differences were observed at the transcriptome level. In the R treatment, among 195 DEGs, the exocarp was accompanied by the formation of a suberin lamellae on the epidermis, with the most dramatic changes seen in gene ontology related to cutin, suberin, and wax biosynthesis, fatty acid metabolism, phenylpropanoid biosynthesis, water channel transporter activity-related russet, and ATP-binding cassette (ABC) transporters (Fig. 3D and Table S5). More specifically, an increased suberin deposition was supported by a consistently higher expression of transcriptsPbHHT1 (103938506) and PbHHT2 (103966555), which increased 11.11-fold and 23.78-fold in russet pear, respectively, and 6.67-fold and 6.64-fold in semi-russet pear, respectively, under R treatment (Fig. 3E-3). Suberin is a biopolyester with glyceride-phenols as its basic unit. The transcripts of PbGPAT5 (glycerol-3-phoshate acyltransferase 5; 103942961) and PbFAR3 (fatty acyl-CoA reductase; 103931004), which are the main synthesizer genes of the enzyme glycerate, were significantly upregulated in the R-treated group compared to expression in the C-treated group, in russet and semi-russet exocarp. In the R-treated group, expression was significantly upregulated in genes involved in fatty acid elongation and metabolism, including PbP450 86A8 (103957382) and PbP450 86B1 (103943216), which encode cytochrome P450 monooxygenase, oxidoreductase, and fatty acid elongase (FAE) in the semi-russet exocarp. $\beta$-keto ester CoA synthetase (KCS) is an integral part of FAE; the genes involved, including PbKCS19 (108865656), PbKCS10 (103949661; 103952998), PbKCS10 (103949661; 103952998), and PbKCS20 (103940571), showed lower expression in russet and semi-russet exocarps. In contrast, PbKCS4 (103966995) and PbKCS11 (103929791) exhibited higher levels in the semi-russet exocarp. $P b C E$ R1 (103929753) was related to wax synthesis gene expression and $P b F A D_{2}(103958977 ; 103964864)$ expression was significantly downregulated in the R-treated group (Fig. 3E-4). ThePbELOVL3 gene is a 
member of the ultra-long-chain fatty acid elongase (ELOVLs) family and was suppressed in the semi-russet exocarp, but its expression was increased in the green exocarp.

In the R-treated group, many lignin monomers were specifically deposited in the secondary cell walls of russet and semi-russet exocarps (Fig. 3E-5). With an increase in lignin, the transcript expression of PbLAC (103929799), Pb CAD1 (103951782), and PbHCT(Shikimic acid/quinic acid hydroxycinnamyl transferase; 103964288) was upregulated in the exocarp of russet and semi-russet pears. The expression of PbCOMT1 (103927980) (2.06-fold), PbCOMT2 (103951572) (5.23-fold), and PbCCoAOMT (103933993) (15.19-folds) was only significantly up-regulated in russet exocarps, whilePbCCR1 (103943280, 108867111) was only upregulated in semi-russet exocarps. There was significant differential expression of $36 \mathrm{PbABC}$ genes. PbABCG6 (103928588, 103936749, 103948344), PbABCG10 (103932334, 103934278), and PbABCG20 homologies (103933813, 103932967) showed increased transcript levels, while PbABCG15(103942099, 103955899, 103963796) and PbABCG11 homologies (103936479, 103930284, 103930593) showed decreased transcript levels in russet and semi-russet exocarps.

In the R-treated group, the exocarp cells not only changed in composition, but also significantly changed their structure (Fig. 3E-3). Genes encoding expansin, a protein that relaxes plant cell walls, including

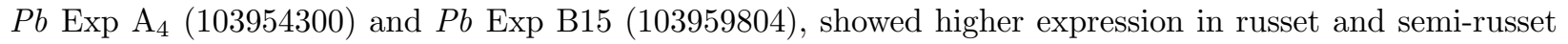
exocarps under R-treatment than in the respective controls. Expansin also significantly interfered with the response to water through activity of the water channel transporter (Fig. 3E-1). Three DPGs ( Pb HVA22C, ERF053, and DHN3) were revealed in the response to water pathway. Three DPGs (aquaporin TIP1-3, aquaporin PIP2-2, and aquaporin PIP2-5) were upregulated in the exocarp of russet pears, while DHN3 and TIP1-3 were significantly downregulated in green exocarps.

\section{iTRAQ and PRM proteome analysis}

Proteome analysis was performed with the same tissues used for the metabolome and transcriptome analyses. Among all cultivars and treatment groups, 85, 121, and 27 DEPs were identified (Fig. 4A-C). GO enrichment analysis of the differential proteins based on the Blast 2 GO results showed that the response to stimulus (64, 42, and 18 proteins identified in CG, ZS, and CY, respectively), single-organism process (83, 61, and 21 , respectively), cellular process $(97,68$, and 22 , respectively), and metabolic process (96, 67, and 20, respectively) played the most important roles in russeting (Fig. 4D-F). There were 29 DEPs that could be confidently related to the response to water and the russeting biosynthesis process (Table S6). Over 30\% of DEPs were related to the response to water, followed by proteins in the plasma membrane. Furthermore, we quantitatively assessed the relative expression of DEPs and DEGs. The relative expression levels of 5 DEPs (SHM1, nLTP3, nLTP4, CYP704C1, HHT1) and 3 DEGs (PODA2, TIP1-3) were consistent with the absolute quantitative expression by PRM (Fig. 4G).

\section{Integrative analysis of metabolome, proteome, and transcriptome}

Cultivars with increased suberin lamellae (russet and semi-russet pears) had significant changes in the geneto-protein and metabolite pathways associated with cutin, suberin, and wax biosynthesis. The fatty acid elongation pathway associated with cork and stratum corneum also underwent significant changes in the semi-russet exocarp (Table 1). We converted and mapped the genes, proteins, and metabolite IDs into the KEGG database and integrated the three sets of data, from which network maps and a mechanism map related to russet color accumulation of the pear exocarp were produced (Fig. 5). Based on statistical data, an interaction network diagram of the metabolites, metabolism-related enzymes, DEGs, DEPs, and DEMs was constructed and centered on the secondary metabolic pathways in all types of pears (Fig. 5A-5C). The 103 DEGs and 18 DEPs were grouped into five clusters (I-V), the groups showed higher connectivity between the DEGs and DEPs in clusters I-IV, and cluster V showed connectivity only in the semi-russet pear. Among the three colored cultivars, there were more connected nodes of clusters I-V in russet and semi-russet pears than in the green pear. There was no direct correlation between transcripts and proteins, but the four genes and their encoded proteins were consistent (Table 2).

\section{Discussion}


Exocarp russeting is controlled by both genetics and environmental effects, with russet depth being primarily due to environmental impact. Despite the histochemical characterizations made in recent research, the biological function of russeting in sand pear exocarp is still unclear regarding defense and stress tolerance (Wang et al., 2016). In the present study, we revealed the mechanism of russeting in the sand pear pericarp by combining transcriptomics, proteomics, and metabolomics analyses for the first time in the literature. Briefly, a series of DEGs were identified and the overlapped DEGs were primarily enriched in the biological processes of wounding, response to stimulus and stress, as well as the secondary metabolic pathway. However, transcriptome and proteome changes are not necessarily directly associated with phenotypic changes (Sumner et al., 2003). Metabolites can more directly reveal the cause of tissue structure formation (Fiehn et al.,2000). Through metabolome profiling, data showed that the overlapped DEMs of lutein had the KEGG ID and were enriched in carotenoid biosynthesis. Lutein is a xanthophyll present in higher plants, microalgae, and cyanobacteria that typically functions to protect the photosystems from oxidative damage during high light stress (Chenet al., 2019). Previous findings reported changes of lutein in plants under different types of stress (Li et al., 2017). Additionally, Huang et al (2014) reported that secondary metabolites exist widely in the phloem of potatoes and that secondary metabolites were identified as potential biomarkers for distinguishing cultivars with russeting features.

\section{Changes in microstructure and chemical composition by water stress}

In the R-treated group, as the russet aggregated, the primary components of the suberin lamellae (suberin, waxes, lignin, and phenolics) changed significantly in russet and semi-russet pears. Researchers found similar results when comparing russet pears with their buds, which did not exhibit russet (Heng et al., 2014; Wang et al., 2014). Similar to the waxy components of 'Pingguoli' (Pyrus bretschneideri Rehd.), the three types of pear exocarp in this study also included unsaturated fatty acids, glycerides, terpenes, alkanes, fatty alcohols, and alkanoic acids. Under $\mathrm{R}$ treatment, the proportion of these compounds were changed. $\mathrm{C}_{18}$ unsaturated fatty acids were abundant, and the content of VLCFAs $\left(\mathrm{C}_{20}-\mathrm{C}_{24}\right)$ was significantly higher in the russet exocarp, which was consistent with the findings of Molina et al. (2006), in which the carbon chain length of the suberin monomer was primarily found to be $\mathrm{C}_{16} \mathrm{C}_{26}$. Graca and Santos (2010) showed that the components of suberin were variable among different species, but similar $\mathrm{C}_{18}$ unsaturated fatty acids were found in the cork-rich exocarp of Quercus variabilisBlume, Picea fortunei (Pseudotsuga sinensis Dode), and potato (Solanum tuberosum L.), which were similar to the pear exocarp.

\section{Lignin biosynthesis}

In the R-treated group, PbHCT , PbCAD1 , PbCOMT1 ,PbCOMT2 , PbCCoAOMT , PbLAC , and PbLAC $(P O D)$, which are genes encoding key downstream proteins in the pathway of phenylpropane metabolism to lignin monomer synthesis, exhibited higher expression. Similarly, the expression of POD enzymes (Q9FLC0, Q42578) was also significantly increased in russet and semi-russet exocarp. The expression of PbCAD6 and $\mathrm{PbCCR}$ 1-like isoform X2 was severely suppressed in the russet exocarp. Bernards (2002) proposed that suppressing the activities of CAD and CCR could decrease the lignin content and lead to cell wall loosening. Kaur et al. (2012) speculated that there is an independent CAD pathway in plants activated by environmental stress and that stress effects on lignin biosynthesis may be present in plants. We found that the expression levels of $P b C A D 1$ were positively correlated with lignin synthesis in the russet exocarp, while the expression levels of $P b C A D 6$ and $P b C C R 1$-like isoform X2 were negatively correlated with lignin synthesis. We speculate that two metabolic branches exist in the CCR and CAD nodes of synthetic lignin monomers and that $\mathrm{PbCAD} 1$ may attribute to lignin monomers, while PbCAD6 may participate in loosening the lignin structure; however, these findings require further study. PbALDH2C4 and the $\beta$-glucanase gene PbBGLU44 were also significantly upregulated in the russet exocarp with russet-enhancing. Moreover, both BGLU45 and BGLU46 are expressed strongly in major organs of lignin deposition in Arabidopsis thaliana (Escamilla-Treviño et al., 2006).

\section{Suberin, cutin, and wax biosynthesis}

The expression of the fatty aldehyde decarbonylase gene, PbCER1, was significantly downregulated. 
PbKCS19 , PbKCS10 ,PbKCS10, and PbKCS20, which all participate in FAE (fatty acid extension), were all inhibited in the russet exocarp under $\mathrm{R}$ treatment. This result is consistent with the findings of Lee (2010). The expression levels of $P b G P A T 5$, PbCYP86A1 , and $P b C Y P 86 B 1$ were significantly upregulated in CG-CL and ZS-CL. Suberin-associated GPAT5 can accommodate a broad range of chain lengths for oxidized and unsubstituted acyl-CoAs and are considered major determinants of cutin and suberin composition (Yang et al.,2012). The CPY86 subfamily includes an important enzyme that catalyzes the $\omega$-hydroxylation of $\mathrm{C}_{16}$ and $\mathrm{C}_{18}$ fatty acids in plants (Benveniste et al., 1998). The increase of oleic acid $\left(\mathrm{C}_{16: 0}\right)$ and palmitic acid $\left(\mathrm{C}_{18: 1}\right)$ in the suberin tissue confirm the biological function of CYP86. Previous studies have shown that PbABCG6 and PbABCG20 may be involved in suberin formation (Hou et al., 2018). PbABCG15 participates in pollen exine development in rice and is also used in the formation of the lipidic cuticle, indicating that it may contribute to an increase in suberin in the exocarp of pears (Wu et al., 2014). Notably, in this study, $P b A B C G 6$ and $P b A B C G 11$ were highly expressed in all seven of the stages analyzed for russet and semi-russet pears, particularly so in the exocarp of pear fruit, where suberin formation occurs. Additionally, PbHHT1 (103966555) and its encoded protein HHT1 (694406379) were found at both the protein level and the transcriptome level, indicating that this gene positively promotes suberin biosynthesis in russeting of pears.

\section{Stress response and water channel and transmembrane transporter activity}

Stress response genes and proteins were involved in the russeting of pear exocarp under rainwater stress. We detected more aquaporins; TIP1-3, aquaporin TIP2-1, and aquaporin PIP2-5 were upregulated in the exocarp of russet and semi-russet pears under R treatment. Studies on the regulatory functions of PIPs and TIPs have speculated that they regulate the transmembrane transport of water through transcription, thereby maintaining water balance in plants under stress (Shao et al ., 2008). The surface accumulation of suberin lamellae (russet) in sand pear exocarps would affect the transpiration rate, as rain and high-humidity conditions promote the regulation of aquaporins, which are presumed to facilitate the expelling of excess water from tissues. In the R-treated group, the exocarp cells of pears not only changed in composition, but also significantly changed structurally. Studies have provided evidence that expansins are associated with environmental stress tolerance in plants (Marowa et al ., 2016). The expression of PbEXPB3b and PbEXP $4 a$ enhanced during russeting stage in Dangshansuli (Pyrus bretschneideri Rehd.) (Heng et al ., 2017). The DEPs of expansin provide more direct evidence of the loosening of the exocarp in russet pears. This also provides strong molecular evidence that mechanical rupture of the cuticle is the motivity of cork layer formation.

In this study, we provided a mechanism model of russeting in exocarp by combining multi-omics data. Briefly, the cork layer serves as a protective barrier after the stratum corneum is damaged; it is formed by the polymerization of multiple complex biosynthetic pathway products. The phenylpropane synthesis pathway forms aromatic compounds such as phenols and lignin monomers. Long-chain fatty acids (mainly $\mathrm{C}_{16}$ and $\mathrm{C}_{18}$ ) are formed by $\omega$-hydroxylation and then extended, oxidized, reduced, and esterified by fatty acyl groups. Glycerin-ester, which is the basic component of the polyester structure, is introduced into the polyester structure by ferulyltransferase HHT to form a polyphenol structure. Polyphenol and polyester are then assembled and transported in the cell wall and sedimentation forms a cormorant. ABC transporters and lipid transfer proteins (LTPs) are the primary transmucosal transport proteins of cutin, suberin, and waxes. A comprehensive diagram of the mechanisms involved in cutin, suberin, and wax in pears is shown in Fig. 5D. The network map indicates that the suberin lamellae deposit process in two russet sand pears may be different. In the semi-russet exocarp under R-treatment, russeting was activated by the PAL-Lignin pathway for lignin biosythesis and the LCFAs-monoacylglyceride for surberin bioythesis. However, in russet exocarp, russet was activated by the PAL-phenols pathway, such as p-cinnamic acid and the BGLU family (coumarine; 3-O-p-trans-Coumaroylalphitolic acid). BGLU44 was upregulated in the phenylpropanoid biosynthesis pathway for coumarine synthesis (Jia et al., 2019). In russet and semi-russet pears, the PpyHHT1 (103966555) gene and encoded protein HHT1 (694406379) enhanced phenol- and ester-polymerization. Conversely, those were suppressed in green pears. Interestingly, triacontaneare is an important component of cutin and wax in the green exocarp and maintains the integrity of the stratum corneum. Deposition of the 
cork layer showed a downward trend in both russet and semi-russet pears. As reported previously, rain and light treatment may affect proteins of PAL, CCR1, CAD, POD, COMT, and HHT1 of phenylalanine-lignin monomer synthesis for russeting in semi-russet pear (Shi et al., 2019a; Shi et al., 2019b). In the present study, russeting is not only caused by the single lignin biological pathway, but also the interaction of suberin, phenylpropane-lignin(phenols), and cutin biosynthesis, in which suberin and lignin are intensified and cutin synthesis is inhibited.

In conclusion, our integrated analysis of the transcriptome, proteome, and metabolome in the exocarp of three different colored pears revealed that rainfall contributes to russeting in pears. In artificial rainfall experiments, a rain-abundant environment led to aggregation of suberin lamellae in the cuticle of the fruit epidermis in russet and semi-russet pears, where the cuticle enacted defenses against biological and abiotic damage. It should be noted that the russet-based phenol-ester defense system led to an alternative to the stratum corneum better adapted to water imbalance.

Our findings present a molecular model of the russeting mechanism under rain-stress of different genotypes of pears. We have already explored the response of russeting under enviroment (rainfall and light) stress in pears, the next task of study would be foucus on biotic stress (disease, insect damage) that may destroy the surface of pear stratum corneum based on global data form multi-omics analysis, need explored to answer how plants integrate multiple stress signals and respond for russeting, which will impact our ability to processing qualities and enhancing resistance to stress in the near future.

\section{Materials and methods}

\section{Plant materials and stress treatments}

In order to explore the possible defense mechanisms of pear against rain, the pear cultivars: russet 'Zao sheng xin shui' (ZS), semi-russet 'Cui guan' (CG), and green 'Cuiyu' (CG) were grown in the Jinshan fruit experiment station of the Shanghai Academy of Agricultural Sciences (30.7875 N, 121.1438 E), Shanghai county, China in June 2017. On the 10th day of fruit development, all cultivars were divided into six groups: ZS-R, CG-R, and CY-R groups were treated with artificial spray rain water (R); and ZS-C, CG-C, and CY-C groups (as the control groups) were wrapped with transparent nano-pore bags to avoid contact with rain (C). Every group consisted of 5 biological replicates, and each replicate had 6 fruits. After 80 days of full bloom (DAFB), whole exocarp slices of each fruit were collected and immediately frozen in liquid nitrogen, then stored at $-80^{\circ} \mathrm{C}$ for RNA, protein, and metabolite extraction.

\section{Electron microscopy detection}

Characteristics of suberin layer formation in the exocarp of fresh pear were observed using a stereoscopic microscope (magnification 10×). Samples were randomly excised from the middle of the pear fruit with 10 replicates per treatment. In brief, the exocarp skin $(5 \mathrm{~mm} \times 5 \mathrm{~mm})$ was excised, frozen in a pre-cooled exocarp tissue holder with frozen glue, and then inserted into a cryogenic liquid nitrogen slurry at a temperature lower than -143.15degC for rapid freezing. Subsequently, the samples were fixed using the quorum 2100 scanning electron microscope cryoscope (SEM; Quorum, England).

\section{Untargeted metabolomics analysis by LC-MS and GC-MS}

The exocarp slices of three pear varieties (R; C) at 80 DAFB were selected to be used in the metabolomics analysis. Analysis was performed using eight biological replicates.

For LC-MS analysis, $60 \mathrm{mg}$ samples were used and the quality control sample (QC) was prepared by mixing the extraction liquid of all samples in equal volume. An ACQUITY UHPLC system (Waters Corporation, Milford, USA) coupled with an AB SCIEX Triple TOF 5600 System (AB SCIEX, Framingham, MA) was used to analyze the metabolic profiling in both ESI positive and ESI negative ion modes. An ACQUITY UPLC BEH C18 column $(1.7 \mu \mathrm{m}, 2.1 \times 100 \mathrm{~mm})$ was employed in both positive and negative modes. The QCs were injected at regular intervals (every 10 samples) throughout the analytical run to provide a set of data from which repeatability could be assessed. In positive ion mode, 5291 metabolite ions were acquired 
and in negative ion mode, 5613 metabolite ions were acquired. Metabolite labeling was completed using the Human Metabolome Database and LIPID MAPS@ Structure Database (LMSD) (Scandiani et al., 2015).

For GC-MS analysis, a $60 \mathrm{mg}$ sample placed in a $1.5 \mathrm{~mL}$ centrifuge tube. The analytical instrument used in this experiment was the 7890B-5977A GC/MSD GC/MSD (Agilent Technologies Inc. CA, USA). A DB-5MS capillary column $(30 \mathrm{~m} \times 0.25 \mathrm{~mm} \times 0.25 \mu \mathrm{m}$; Agilent J \& W Scientific, Folsom, CA, USA $)$ with a carrier gas of high-purity helium (purity no less than $99.999 \%$ ), flow rate $1.0 \mathrm{~mL} / \mathrm{min}$, and inlet temperature of $260^{\circ} \mathrm{C}$ was used. Metabolites were quantified using the NIST and Fiehn database. A total of 256 metabolites were detected.

For the identification of differentially expressed metabolites (DEMs), unsupervised principal component analysis (PCA) was used to observe the overall distribution among samples and the stability of the whole analytical process. Supervised projection to latent structure-discriminant analyses (PLS-DA) were carried out to distinguish the overall differences among groups. A variable important for the projection (VIP) $>1$ and $\mathrm{P}$-value $<0.05$ were considered as threshold values for the differentially expressed metabolites (DEMs).

\section{Metabolite targeted detection}

The UPLC-Q-TOF/MS analysis method was used to qualitatively and quantitatively detect the target compound (phenolic substances and long-chain/very long fatty acids) in the exocarp samples. A UPLC-QTOF/MS mass spectrometry system (Waters, USA) equipped with an electrospray (ESI) ion source and UNIFI 1.8.1 workstation were used.

\section{RNA-seq analysis}

Total RNA was extracted from exocarp slices and library preparation was performed according to Illumina standard instructions (TruSeq Stranded RNA LT Guide). The raw reads were filtered by Seqtk before mapping to the genome using TopHat (version: 2.0.9) (Trapnell et al., 2009). The gene fragments were counted using HTSeq followed by TMM (trimmed mean of M values) normalization (Anders et al., 2015). Significant differentially expressed genes (DEGs) were identified as those with a false discovery rate (FDR) value above the threshold $(\mathrm{Q}<0.05)$ and fold-change $>2$ using edgeR software (Benjamini et al., 1995). Annotation analysis of DEGs was performed by gene ontology (GO) (Ashburner et al., 2000) using the Kyoto Encyclopedia of Genes and Genomes (KEGG) enrichment (Kanehisa et al., 2000).

\section{Proteome with iTRAQ, LC-MS/MS, and data analysis}

The exocarp of three pear varieties pear under rain-abundance and control conditions were used with two biological replicates. Protein was extracted from tissues samples as described by Daqiu et al (2015). Protein digestion was carried out following the FASP procedure as previously described. After digestion with trypsin (protein: trypsin, 30:1) overnight at 37degC, the peptides were desalted and labeled with the 8-plex iTRAQ reagent (AB SCIEX, Framingham, MA, USA), according to the FASP's protocols for iTRAQ labelling (Wiśniewski et al., 2009). iTRAQ reagents had molecular masses of 113 (CG-R), 114 (CG-C), 115 (ZS-R), 116 (ZS-C), 117 (CY-R), 118 (CY-C) and 119 (Mixture) Da, respectively.

For LC-MS/MS analysis, the parameters and methods were carried out as described by Fu et al. (2015). Subsequently, the MS/MS data were analyzed for protein identification and quantification using Protein Pilot Software v.5.0 (Sciex Inc., USA). Only proteins identified at global FDR [?] 1\% and unique peptides [?] 1 were considered for the protein lists and further downstream analysis. The differentially expressed proteins (DEP) were identified with p-values $<0.05$ and fold change [?]1.2. In addition, the experimental data was analyzed using Proteome Discoverer TM 22 (Thermo Corporation, USA) software and the NCBI Pear Database. The identified DEPs were annotated with GO terms and KEGG pathways enrichment analysis.

\section{Target analysis by parallel reaction monitoring (PRM)}

Targeted quantification and verification were carried out in 6 samples. The sample preparation for PRM was completed using the method outlined by Martinez (2017). Proteins were digested and desalted by an 
C18-Reverse-Phase SPE Column and the iRT standard peptides were dissolved by a relative buffer according to the user manual (2xiRT Kit Quick Reference Card). PRM analysis was performed with a Q-Exactive Plus mass spectrometer (Thermo Fisher Scientific, San Jose, CA). The original offline files were imported into Proteome Discover 2.3 software for the database (LM07171185NCBI-Pyrus pear. fasta) search. Using Skyline software, the target peptide lists were selected from the DDA results and then imported into the inclusion list of the Xcalibur PRM method editing module. The search results were imported into the Skyline software (MacLean et al ., 2010) and the actual detection retention time and relative quantification values of the iRT standard peptides were derived (Table S10) and combined with statistics from Excel, the results from the Graphpad Prism 8.0 software, the retention time distribution, and the correlation with the theoretical retention time. All the standard peptides (11/11) were detected (Fig. S2), and the retention time deviations between the components were small. The correlation coefficient $\left(\mathrm{R}^{2}\right)$ between detection value and retention time was 0.9986, indicating that qualitative accuracy was reliable (Fig. S3).

\section{Quantitative real-time PCR}

Total RNA was extracted from the pear exocarp tissue using the Plant RNA Kit (Omega Bio-Tek, Guangzhou, China). The cDNA was reverse transcribed using a Revert Aid First Strand cDNA Synthesis kit (Takara RRO47).

\section{Integration analysis of transcriptome, proteome, and metabolome profiles}

In order to explore the key genes, proteins, and metabolites, the common or unique pathways of the transcriptome, proteome, and metabolome groups were integrated and analyzed. ID conversion was used to convert genes, proteins, and metabolite IDs into KEGG format (Tables S7, S8, and S9). The IDs were then simultaneously mapped to the KEGG database through GENMAPP to obtain the pathway of simultaneous participation and visualize the corresponding pathway graph. Protein-pathway and protein-protein correlations were obtained based on integrated analysis and then network maps were drawn using Cytoscape 3.6.1. (Table S2).

\section{Statistical analyses}

The results of lignin content and the quantitative expression of DEGs, DEPs, and DEMs are presented as the mean \pm standard deviation (SD) and were analyzed by univariate statistical analysis. Significant differences were analyzed using a one-way analysis of variance (ANOVA) and least significant difference (LSD) multiple range tests using SPSS 17.0 software (IBM, Armonk, NY, USA). A p $<0.01$ indicated a statistically significant difference between treatments.

\section{Conflict of interest}

No conflict of interest exits in the submission of this manuscript, and manuscript is approved by all authors for publication. I would like to declare on behalf of my co-authors that the work described was original research that has not been published previously, and not under consideration for publication elsewhere, in whole or in part. All the authors listed have approved the manuscript that is enclosed.

\section{Author contributions}

Conception and design of the research: JL, YZ, CS; acquisition of data: CS; analysis and interpretation of data: CS; statistical analysis: CS; obtaining funding: JL; drafting the manuscript: CS; revision of manuscript for important intellectual content: CS; measured the russet index, and RNA extracting: XW. All authors read and approved the final manuscript.

\section{Acknowledgements}

This study was financially supported by Research and Demonstration of High-efficiency Production Technology in Green Pear Orchard (SAS2019 1-3). In addition, we thank the anonymous reviewers for their valuable comments regarding the manuscript.

\section{References}


Anders S, Pyl PT, Huber W. (2015). HTSeq-a Python framework to work with high-throughput sequencing data. Bioinformatics 31 : 166-169.

Ashburner M, Ball CA, Blake JA, Botstein D, Cherry JM. (2000). Gene ontology: Tool for the unification of biology. Nature genetics $25: 25-29$.

Benjamini Y, Hochberg Y. (1995). Controlling the false discovery rate: A practical and powerful approach to multiple testing. J Roy Statist Soc Ser B $5 \mathbf{7}: 289-300$.

Benveniste I, Tijet N, Adas F, Philipps G, Salaün J-P, Durst F.(1998). Cyp86a1 from Arabidopsis thaliana encodes a cytochrome p450-dependent fatty acid omega-hydroxylase. Biochem Biophys Res Commun 243 : 688-693.

Bernards MA. (2002). Demystifying suberin. Canadian Journal of Botany 80 : 227-240.

Chen JH, Chen CY, Hasunuma T, Kondo A, Chang CH, Ng IS, Chang JS. (2019). Enhancing lutein production with mixotrophic cultivation of Chlorella sorokiniana MB-1-M12 using different bioprocess operation strategies. Bioresource Technology 278 : 17-25.

Cohen H, Szymanski J, Aharoni A. (2017). Assimilation of 'omics' strategies to study the cuticle layer and suberin lamellae in plants. Journal of Experimental Botany 68 (19): 5389-5400.

Daqiu Z, Saijie G, Zhaojun H, Jiasong M, Jun T. (2015). Quantitative Proteomics Analysis of Herbaceous Peony in Response to Paclobutrazol Inhibition of Lateral Branching. International journal of molecular sciences 16 : 24332-24352.

Escamilla-Treviño LL, Chen W, Card ML, Shih MC, Cheng CL, Poulton JE. (2006). Arabidopsis thaliana $\beta$-Glucosidases BGLU45 and BGLU46 hydrolyse monolignol glucosides. Phytochemistry 67 : 16511660 .

Fiehn O, Kopka J, Dörmann P, Altmann T, Trethewey RN, Willmitzer L. (2000). Metabolite profiling for plant functional genomics. Nature Biotechnology 18 : 1157-1161.

Franke R, Schreiber L. (2007). Suberin-a biopolyester forming apoplastic plant interfaces. Current Opinion in Plant Biology 10 : 252-259.

Fu Y, Zhang H, Mandal SN, Wang C, Ji W. (2015). Quantitative proteomics reveals the central changes of wheat in response to powdery mildew. Journal of Proteomics 130 : 108-119.

Gamble J, Jaeger SR, Harker FR. (2006). Preferences in pear appearance and response to novelty among Australian and New Zealand consumers. Postharvest Biol Technol 41 : 38-47.

Graça J, Santos S. (2007). Suberin: A biopolyester of plants' skin. Macromolecular Bioscience . 7, 128-135.

Heng W, Liu L, Wang MD, Jia B, Liu P, Ye ZF, Zhu LW. (2014). Differentially expressed genes related to the formation of russet fruit skin in a mutant of 'Dangshansuli' pear (Pyrus bretchnederirehd) determined by suppression subtractive hybridization. Euphytica196 : 285-297.

Heng W, Wang Z, Jiang X, Jia B, Liu P, Liu L, Ye ZF, Zhu LW.(2016). The role of polyamines during exocarp formation in a russet mutant of 'Dangshansuli' pear (Pyrus bretschneideri Rehd.).Plant Cell Reports 35 : 1841-1852.

Heng W, Yang J, Hou Z, Li F, Jia B, Liu P, Ye ZF, Zhu LW.(2017). Characterization and expression analysis of PbEXP genes in the epidermis of pear (Pyrus bretschneideri rehd). Plant Growth Regulation $\mathbf{8 4}$ $: 1-9$.

Hou Z, Jia B, Li F, Liu P, Liu LY, Zhen FZ, Li WW, Qi H, Heng W. (2018). Characterization and expression of the ABC family (G group) in 'Dangshansuli' pear (Pyrus bretschneideri Rehd.) and its russet mutant. Genetics and Molecular Biology 41 : 137-144. 
Huang WL, Serra O, Dastmalchi K, Jin LQ, Yang LJ, Stark RE.(2014). Deconstructing a Plant Macromolecular Assembly: Chemical Architecture, Molecular Flexibility, And Mechanical Performance of Natural and Engineered Potato Suberins Biomacromolecules15 :799-811.

Inoue E, Kasumi M, Sakuma F, Anzai H, Amano K, Hara H. (2006). Identification of RAPD marker linked to fruit skin color in Japanese pear (Pyrus pyrifolia Nakai). Scientia Horticulturae107 : 254-258.

Jia X-M, Zhu Y-F, Hu Y, Zhang R, Cheng L, Zhu Z-L, Zhao T, Zhang X, Wang Y-X. (2019). Integrated physiologic, proteomic, and metabolomic analyses of Malus halliana adaptation to saline-alkali stress. Hortic Res $\mathbf{9 1}$.

Kanehisa M, Goto S. (2000). KEGG: Kyoto Encyclopedia of Genes and Genomes. Nucleic Acids Research $28: 27-30$.

Kaur H, Shaker K, Heinzel N, Ralph J, Gális I, Baldwin IT.(2012). Environmental stresses of field growth allow cinnamyl alcohol dehydrogenase-deficient Nicotiana attenuata plants to compensate for their structural deficiencies. Plant Physiol 159 : 1545-1570.

Lara I, Belge B, Goulao LF. (2015). A focus on the biosynthesis and composition of cuticle in fruits. $J$ Agric Food Chem63 : 4005-4019.

Lashbrooke J, Aharoni A, Costa F. (2015). Genome investigation suggests MdSHN3, an APETALA2domain transcription factor gene, to be a positive regulator of apple fruit cuticle formation and an inhibitor of russet development. Journal of Experimental Botany 66 : 6579-6589.

Lee SB, Jung SJ, Go YS, Kim HU, Kim JK, Cho HJ, Park OK, Suh MC. (2010). Two Arabidopsis 3-ketoacyl CoA synthase genes, KCS20 and KCS2/DAISY, are functionally redundant in cuticular wax and root suberin biosynthesis, but differentially controlled by osmotic stress.Plant Journal 60 : 462-475.

Legay S, Guerriero G, Deleruelle A, Lateur M, Evers D, André CM, Hausman JF (2015) Apple russeting as seen through the RNA-seq lens: Strong alterations in the exocarp cell wall. Plant Molecular Biology 88 : 21-40.

Li R, Kang C, Song X, Yu L, Liu D, He S, Zhai H, Liu Q. (2017). A ל-carotene desaturase gene, IbZDS, increases $\beta$-carotene and lutein contents and enhances salt tolerance in transgenic sweet potato.Plant Science 262 : 39-51.

MacLean B, Tomazela DM, Shulman N, Chambers M, Finney GL, Frewen B, Kern R, Tabb DL, Liebler DC, MacCoss MJ. (2010). Skyline: An open source document editor for creating and analyzing targeted proteomics experiments. Bioinformatics 26 (7): 966-968.

Markus R, Lukas S. (2001). Protecting against water loss: Analysis of the barrier properties of plant cuticles. Journal of Experimental Botany 52 : 2023-2032.

Marowa P, Ding A, Kong Y. (2016). Expansins: Roles in plant growth and potential applications in crop improvement. Plant Cell Reports 35 : 949-965.

Martinez E, Lesur A, Devis L, Cabrera S, Matias-Guiu X, Hirschfeld M, Asberger J, Van Oostrum J, Casares de Cal MLÁ, Gómez-Tato A, Reventos J, Domon B, Colas E, Gil-Moreno A. (2017). Targeted proteomics identifies proteomic signatures in liquid-biopsies of the endometrium to diagnose endometrial cancer and assist in the prediction of the optimal surgical treatment. Clinical Cancer Research 23 : 6458-6467.

Miao M, Li L, Wang Y. (2018). A targeted proteomic approach for heat shock proteins reveals DNAJB4 as a suppressor for melanoma metastasis. Analytical Chemistry 90 : 6835-6842.

Molina I, Bonaventure G, Ohlrogge J, Pollard M. (2006). The lipid polyester composition of Arabidopsis thaliana andBrassica napus seeds. Phytochemistry 67 : 2597-2610. 
Pollard M, Beisson F, Li Y, Ohlrogge JB. (2008). Building lipid barriers: Biosynthesis of cutin and suberin. Trends in Plant Science 13 : 236-246.

Sumner LW, Mendes P, Dixon RA. (2003). Plant metabolomics: Large-scale phytochemistry in the functional genomics era.Phytochemistry $62: 817-836$.

Scandiani MM, Luque AG, Razori MV, Ciancio Casalini L, Aoki T, O'Donnell K, Cervigni GD, Spampinato CP. (2015). Metabolic profiles of soybean roots during early stages of Fusarium tucumaniaeinfection. J Exp Bot 66 : 391-402.

Shao HB, Chu LY, Shao MA, Zhao CX. (2008). Advances in functional regulation mechanisms of plant aquaporins: Their diversity, gene expression, localization, structure and roles in plant soil-water relations (Review). Molecular Membrane Biology 25 : 179-191.

Shi CH, Qi BX, Wang XQ, Shen LY, Luo J, Zhang YX. (2019a). Proteomic analysis of the key mechanism of exocarp russet pigmentation of semi-russet pear under rainwater condition. Scientia Horticulturae 254 : 178-186.

Shi CH, Wang XQ, Zhang XY, Shen LY, Luo J, Zhang YX. (2019b). Response of fruit bagging to lignin biosynthesis and expression of related genes in fruit peel of sand pear (Pyrus pyrifolia Nakai) cv. Cuiguan. HortScience 54 :1989-1997.

Sumner LW, Mendes P, Dixon RA. (2003). Plant metabolomics: Large-scale phytochemistry in the functional genomics era.Phytochemistry $62: 817-836$.

Trapnell C, Pachter L, Salzberg SL. (2009). TopHat: Discovering splice junctions with RNA-Seq. Bioinformatics 25 : 1105-1111.

Wang YZ, Dai MS, Cai DY, Zhang S, Shi ZB. (2016). A review for the molecular research of russet/semi-russet of sand pear exocarp and their genetic characters. Scientia Horticulturae 210 : 138-142.

Wang YZ, Dai MS, Zhang SJ, Dai MS. (2014). Exploring candidate genes for exocarp russet pigmentation of sand pear (Pyrus pyrifolia) via RNA-Seq data in two genotypes contrasting for exocarp color. PLOS ONE 85 : 123-134.

Wisniewski JR, Zougman A, Nagaraj N, Mann M. (2009). Universal sample preparation method for proteome analysis. Nature Methods6 : 359-362.

Wu L, Guan Y, Wu Z, Yang K, Lv J, Converse R, Huang Y, Mao J, Zhao Y, Wang Z, Min H, Kan D, Zhang Y. (2014). OsABCG15 encodes a membrane protein that plays an important role in anther cuticle and pollen exine formation in rice. Plant Cell Rep33 :1881-1899.

Yang W, Simpson JP, Li-Beisson Y, Beisson F, Ohlrogge B.(2012). A land-plant-specific glycerol-3phosphate acyltransferase family in Arabidopsis: substrate specificity, sn-2 preference, and evolution. Plant Physiology 160 : 638-652.

Table 1. The three omics common metabolic pathways

\section{Protein}

Semi-russet CG ( $R$ vs $C)$

Biosynthesis of secondary metabolites

Cutin, suberin, and wax biosynthesis

Fatty acid degradation

Russet ZS (R vs C)

Fatty acid elongation

Fatty acid degradation

Cutin, suberin, and wax biosynthesis

Biosynthesis of secondary metabolites

\section{P-value Metabolism}

2.40E-02

$3.12 \mathrm{E}-01$

$4.34 \mathrm{E}-01$

$3.23 \mathrm{E}-02$

$4.32 \mathrm{E}-02$

$1.98 \mathrm{E}-01$

$2.32 \mathrm{E}-01$
Biosynthesis of secondary metabolites Cutin, suberin, and wax biosynthesis Fatty acid degradation

Fatty acid elongation

Fatty acid degradation

Cutin, suberin, and wax biosynthesis

Biosynthesis of secondary metabolites
P-value

$1.68 \mathrm{e}-01$

$1.35 \mathrm{e}-01$

$2.36 \mathrm{e}-01$

$1.07 \mathrm{e}-01$

$1.46 \mathrm{e}-01$

$3.10 \mathrm{e}-03$

$6.28 \mathrm{e}-04$

\section{Transcription}

Biosynthesis of seco Cutin, suberin, and Fatty acid degradat

Fatty acid elongatio Fatty acid degradat Cutin, suberin, and Biosynthesis of seco 
Steroid biosynthesis

Table 2. Top hits common to RNA-seq and iTRAQ proteomics

\begin{tabular}{lllll}
\hline Gene name & Uniprot & Description & $\log 2$ FC RNA & P value \\
Semi-russet CG (R vs C) & & & & \\
PYL6 & Q8S8E3 & major allergen Pru ar 1-like & -4.88 & $1.91 \mathrm{E}-2$ \\
LTP3 & Q42589 & non-specific lipid-transfer protein-like & -3.23 & $2.65 \mathrm{E}-23$ \\
KCS4 & Q9LN49 & 3-ketoacyl-CoA synthase 4-like & -4.34 & $3.78 \mathrm{E}-12$ \\
ALDH2B7 & Q8S528 & aldehyde dehydrogenase family 2 member B7 & 3.15 & $1.25 \mathrm{E}-10$ \\
LTP4 & Q9LLR6 & non-specific lipid-transfer protein & -3.26 & $2.01 \mathrm{E}-12$ \\
HHT1 & Q94CD1 & omega-hydroxypalmitate O-feruloyl transferase-like & 3.21 & $9.39 \mathrm{E}-08$ \\
Russet ZS (R vs C) & & & & $1.77 \mathrm{E}-0$. \\
At3g55800 & P46283 & sedoheptulose-1.7-bisphosphatase. chloroplastic-like & 1.09 & -2.17 \\
LTP3 & Q9LLR7 & non-specific lipid-transfer protein & 1.67 & $2.13 \mathrm{E}-22$ \\
BGLU17 & O64882 & vicianin hydrolase-like & \\
Green CY (R vs C) & & & \\
Q94G9 & FBA2 & fructose-bisphosphate aldolase 1. chloroplastic-like & 2.22 & $5.39 \mathrm{E}-5$ \\
\hline
\end{tabular}

\section{Legends to figures}

Figure 1. Phenotype and physiology changes in the exocarp of three types pear, semi-russet 'Cuiguan' (CG), russet 'Zaoshengxinshui' (ZS), and green 'Cuiyu' (CY) under rain-abundant (R) and control (C) conditions.

1. Morphology of pear exocarp of three varieties under camera;

2. Morphology of pear exocarp of three varieties under scanning electron microscope $(500 \times ; 1500 \times)$;

3. The russet index of ' $\mathbf{Z S}$ ' and 'CG' pears in R treatment was higher than that of the respective control;

4. The cork layer thickness of ' $\mathbf{Z S}$ ' and ' $\mathbf{C G}$ ' pearsafter $\mathrm{R}$ treatment was higher than that of the respective control.

Data are expressed as the mean $\pm \mathrm{SD}$ of three biological replicates. ${ }^{* *} \mathrm{p}<0.01$.

Figure 2. Differential metabolites in the exocarp of three types pear, semi-russet 'Cuiguan' (CG), russet 'Zaoshengxinshui' (ZS), and green 'Cuiyu' (CY) under rain-abundant (R) and control (C) conditions.

1. PCA (Principal Component Analysis) of differentially expressed metabolites among ZS, CG, and CY groups by LC-MS (Liquid chromatography-tandem mass spectrometry);

2. PCA of differentially expressed metabolites among ZS, CG, and CY groups by GC-MS(Gas chromatography-mass spectrometry);

3. Venn diagrams comparing overlap of differentially expressed metabolites among ZS, CG, and CY groups;

4. Heatmap of differential metabolites related to cork layer formation among ZS, CG, and CY groups $(\mathrm{n}=8)$;

5. Absolute quantification of differential metabolites related to the cork layer in the exocarp of ZS, CG, and $\mathrm{CY}$ pears in $\mathrm{R}$ and $\mathrm{C}$ conditions $(\mathrm{n}=9)$.

The data are represented as the mean \pm standard deviation. ${ }^{*} \mathrm{p}<0.05$. ${ }^{* *} \mathrm{p}<0.01$.

Figure 3. Abundance and functional annotation of overlapped differentially expressed genes (DEGs) in the exocarp of three types pear, semi-russet 'Cuiguan' (CG), russet 'Zaoshengxinshui' (ZS), and green 'Cuiyu' $(\mathrm{CY})$ under rain-abundant $(\mathrm{R})$ and control $(\mathrm{C})$ conditions. 
1. Venn diagrams comparing overlap of DEGs;

2. GO (Gene Ontology) annotation of the DEGs in three groups;

3. Bubble chart showing the KEGG (Kyoto Encyclopedia of Genes and Genomes) enrichment pathway of top 20;

4. Heat map of DEGs related to cork layer formation among ZS, CG, and CY groups;

5. Quantitative real-time RT-PCR (qPCR) analysis of genes involved in water response and cork layer formation of $\mathrm{ZS}, \mathrm{CG}$, and $\mathrm{CY}$ pears in $\mathrm{R}$ and $\mathrm{C}$ conditions $(\mathrm{n}=3)$.

1. p-value: The smaller the p-value. the more significant the path enrichment of the corresponding pathway; 2. Number: the number of differential genes/proteins/metabolites enriched on this pathway; 3. Rich factor: rich factor $=$ number of differential genes/proteins/metabolites in this pathway/number of all genes/proteins/metabolites in this pathway. 4. Each value represents the mean \pm standard error (SE) of rain-abundant $(\mathrm{R})$ and control $(\mathrm{C})$ conditions.

Figure 4. Abundance and functional annotation of overlapped differentially expressed proteins (DEPs) in the exocarp of three types pear, semi-russet 'Cuiguan' (CG), russet 'Zaoshengxinshui' (ZS), and green 'Cuiyu' (CY) under rain-abundant (R) and control (C) conditions.

(A)-(C) Volcano plots showing the differential expression of proteins in ZS, CG, and CY;

(D)-(F) GO (Gene Ontology) pathway analysis of overlapped DEPs;

(G) The proteins associated with water response and cork layer in the exocarp of 'ZS', 'CG', and 'CY' pears in $\mathrm{R}$ and $\mathrm{C}$ conditions using parallel reaction monitoring (PRM).

Data are shown as the mean \pm standard deviation. ${ }^{*} \mathrm{p}<0.05 .{ }^{*} \mathrm{p}<0.01$ vs no significant difference by one-way ANOVA (Analysis of Variance) for each condition $(\mathrm{n}=3)$.

Figure 5. Connection network and mechanism model of suberin lamellae formation metabolism and transmembrane transport between regulatory genes, proteins, and cork layer-related metabolites.

The networks in semi-russet 'Cuiguan' (CG), 'Zaoshengxinshui' (ZS), and green 'Cuiyu' (CY) were visualized with the Cytoscape software (version 3.6.1).

(D) The biosynthesis and transmembrane transport of suberin to the cell wall. : Indicates that metabolites involved in the biosynthesis pathway are identified in pears; Blue or red color indicates the strongly enhanced or repressed of DEGs (differentially expressed genes), DEPs (differentially expressed proteins), or DEMs(differentially expressed metabolites) at the transcript, protein, and/or metabolic levels. 1. : Indicates the direction of the pathway. 2. Circular: represents genes (proteins); 3. Triangle arrow: represents metabolic metabolites; 4. Square: represents the signal path (pathway); 5. Map node size indicates degree: low values to small sizes and low values to bright colors. Degree is the most direct measure to describe node centrality in network analysis. The larger the node degree, the higher the centrality degree of the node and the more important the node is in the network.

\section{SUPPORTING INFORMATION}

Additional supporting information may be found in the online version of this article.

Table S1. Different metabolites in the exocarp of three types pear, semi-russet 'Cuiguan' (CG), russet 'Zaoshengxinshui' (ZS), and green 'Cuiyu' (CY) under rain-abundant (R) and control (C) conditions.

Table S2. Overlapped molecular functions of DEGs (differentially expressed genes) identified in the exocarp of three types pear, semi-russet 'Cuiguan' (CG), russet 'Zaoshengxinshui' (ZS), and green 'Cuiyu' (CY) under rain-abundant $(\mathrm{R})$ and control $(\mathrm{C})$ conditions.

Table S3. GO (Gene Ontology) function intersection in the exocarp of three types pear, semi-russet 'Cuiguan' (CG), russet 'Zaoshengxinshui' (ZS), and green 'Cuiyu' (CY) under rain-abundant (R) and control (C) conditions. 
Table S4. DEGs (differentially expressed genes) annotation by KEGG pathways (Top 30) analysis in the exocarp of three types pear, semi-russet ' $\mathrm{CG}$ ', russet 'ZS', and green ' $\mathrm{CY}$ ' under rain-abundant (R) and control $(\mathrm{C})$ conditions.

Table S5. DEGs (differentially expressed genes) most dramatic changes were seen in gene ontology related to cork layer in the exocarp of three types pear, semi-russet 'Cuiguan' (CG), russet 'Zaoshengxinshui' (ZS), and green 'Cuiyu' (CY) under rain-abundant (R) and control (C) conditions.

Table S6. DFPs (differentially expressed proteins) related with russeting biosynthesis by iTRAQ (isobaric tags for relative and absolute quantification).

Note: ${ }^{*}$ p value $<0.05, \ldots$ : upregulated, FC[?]1.2, —: downregulated, FC[?]0.83.

Table S7. ID conversion to convert genes and proteins in semi-russet 'Cuiguan' exocarp under rain-abundant $(\mathrm{R})$ and control $(\mathrm{C})$ conditions.

Table S8. ID conversion to convert genes and proteins in russet 'Zaoshengxinshui'exocarp under rainabundant (R) and control (C) conditions.

Table S9. ID conversion to convert genes and proteins in green exocarp 'Cuiyu' under rain-abundant (R) and control $(\mathrm{C})$ conditions.

Table S10. Retention time of iRT (indexed Retention Time) in DDA(Digital Differential Analyzer).

Figure S1. The russet index and cork layer thickness in the exocarp of three types pear, 'Cuiguan' (CG), russet 'Zaoshengxinshui' (ZS), and green 'Cuiyu' (CY) under rain-abundant (R) and control (C) conditions at different stages of DAFB (Day after flower blooming).

The data are represented as the mean +- standard deviation $(\mathrm{n}=9)$. ${ }^{* *} \mathrm{p}<0.01$.

Figure S2. Eleven standard peptides were detected and retention time between components.

Figure S3. Regression analysis of measured value and theoretical value of iRT (internal standard peptide retention time). 

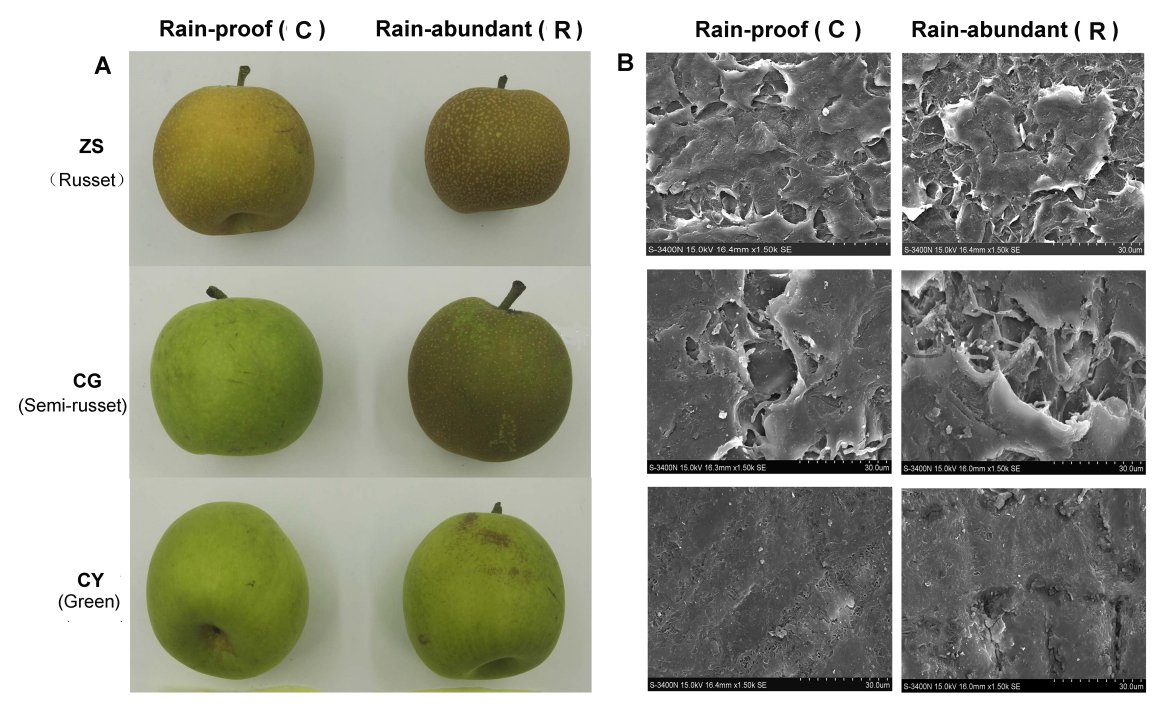

C

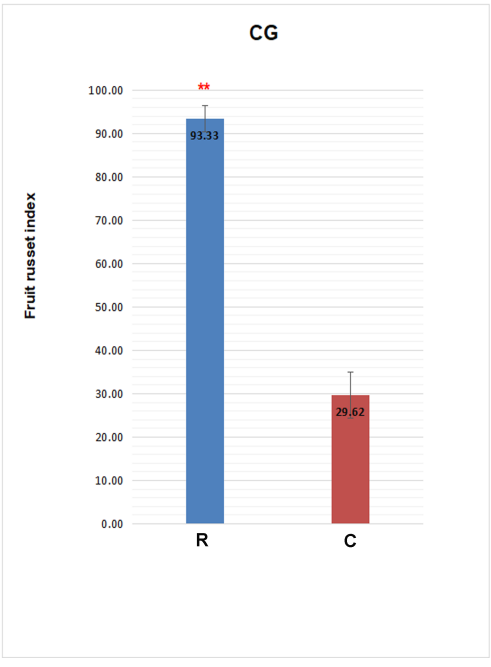

D

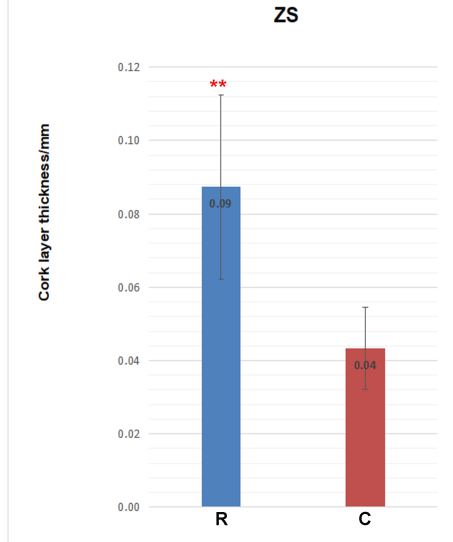

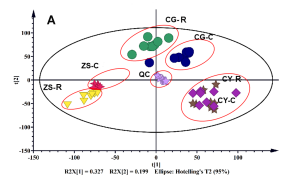

(10)

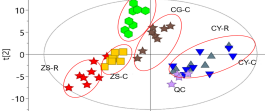

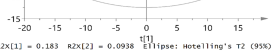
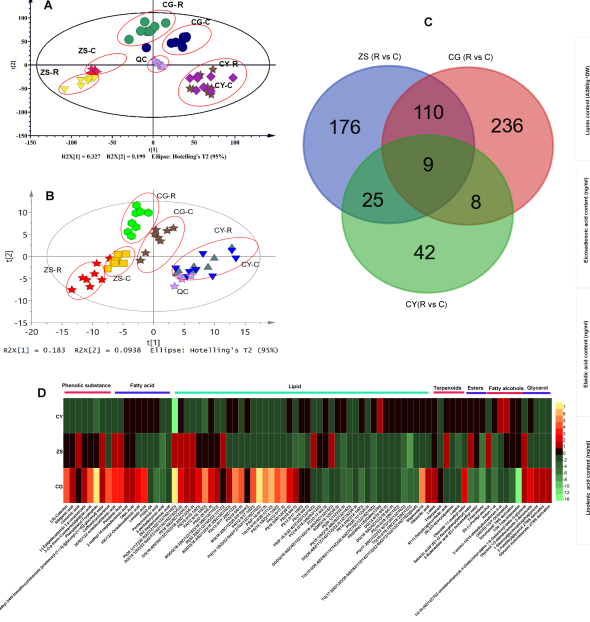

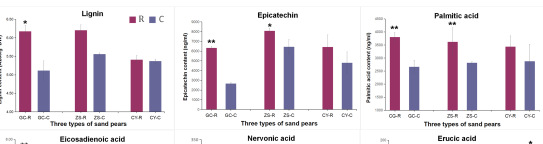

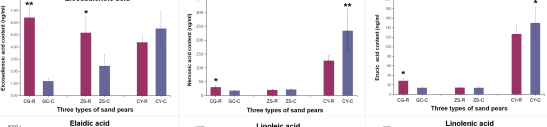

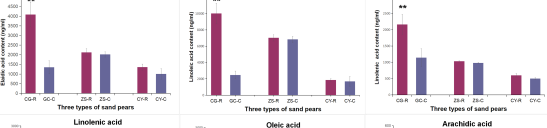

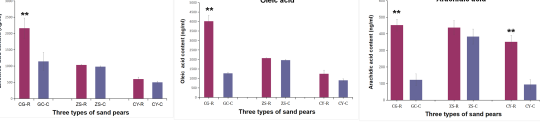




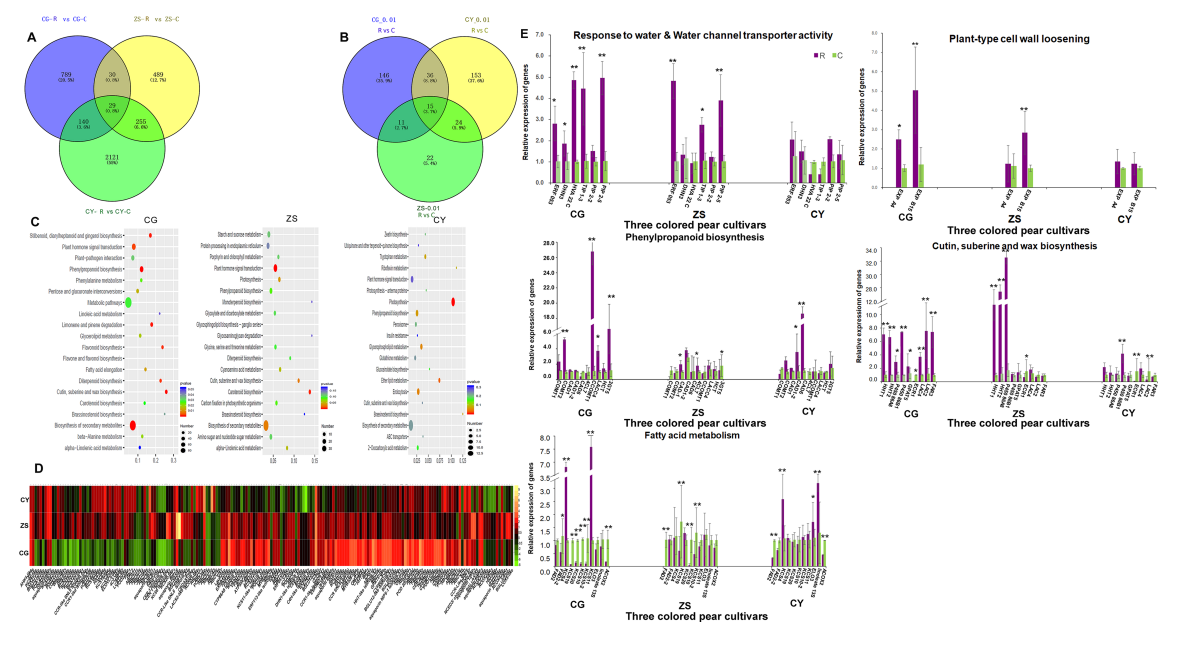



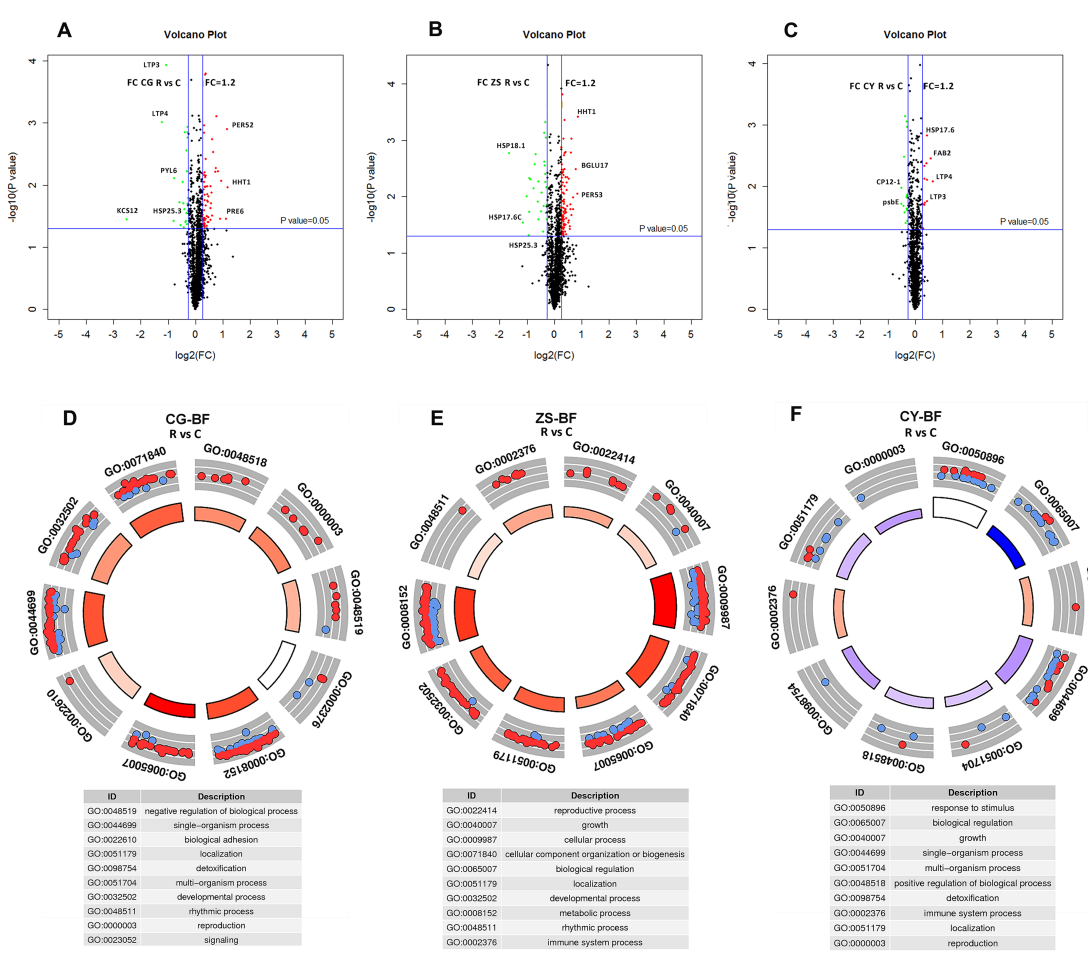

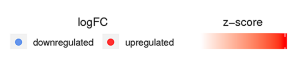

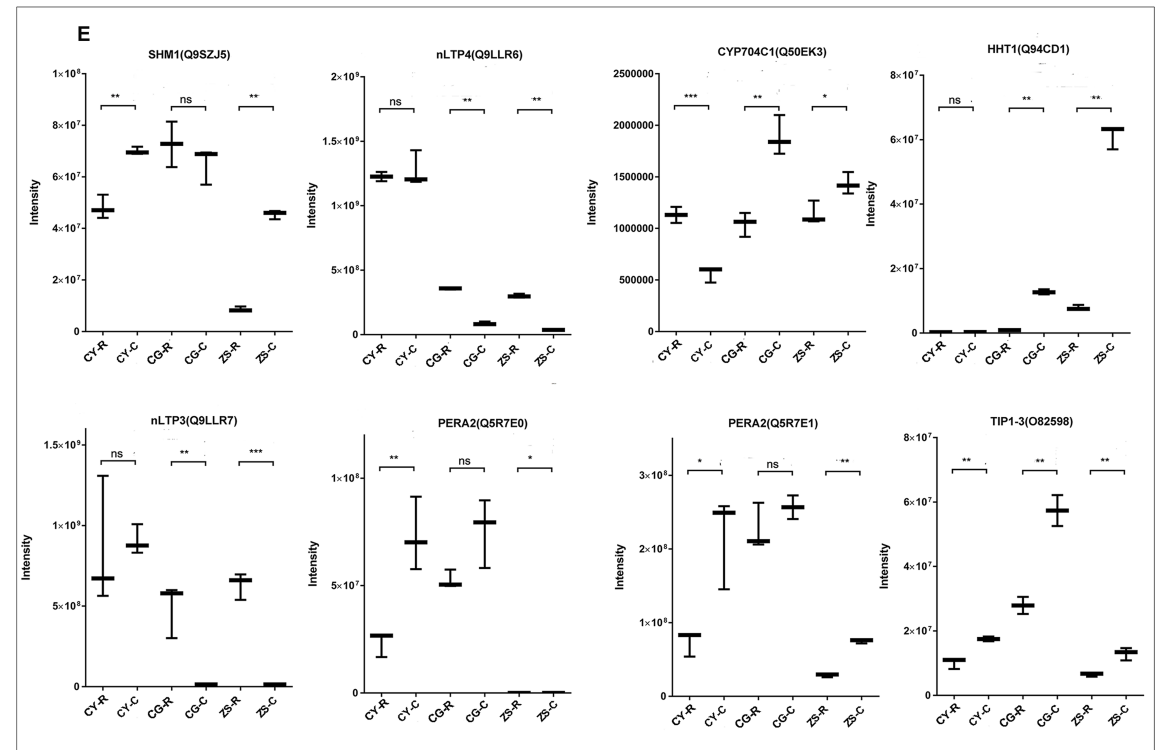




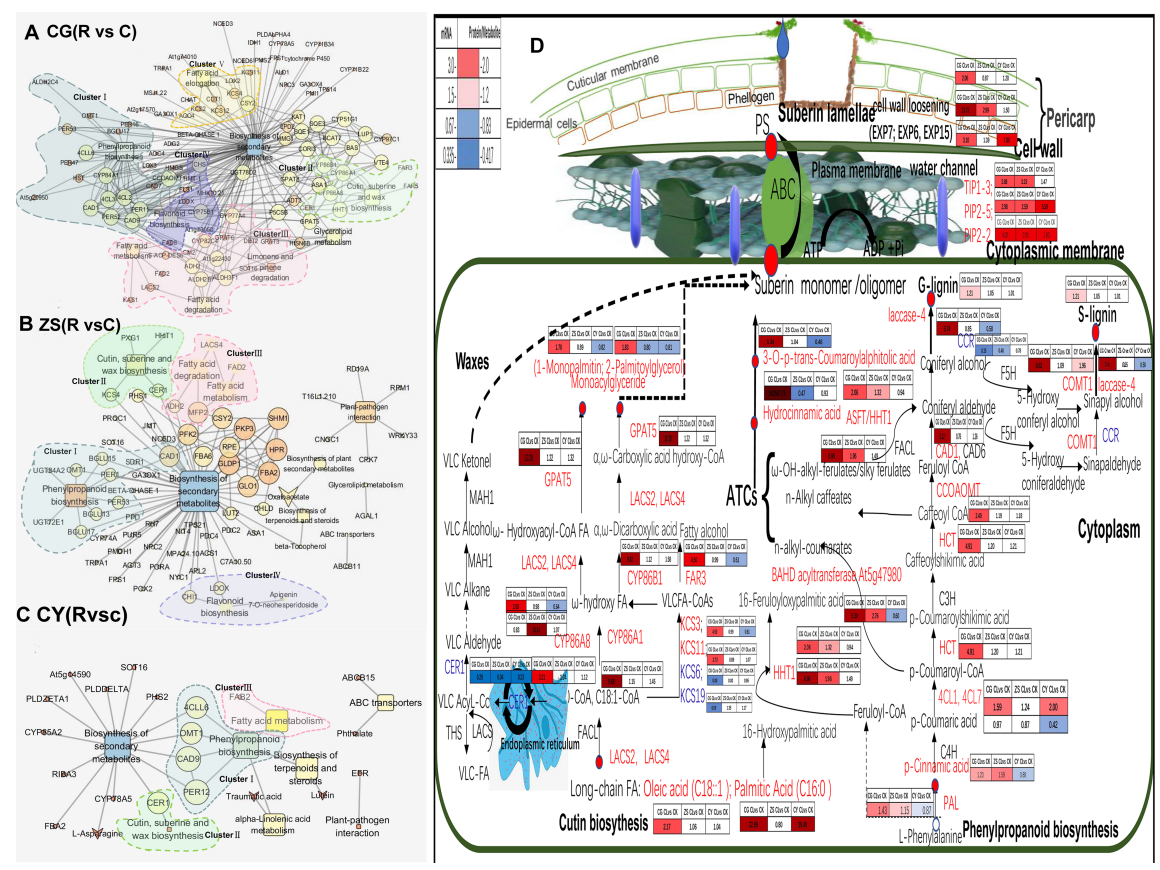

